\title{
An ex vivo Approach to Assess Mitochondrial ROS by Flow Cytometry in AAV-tagged Astrocytes in Adult Mice \\ Carlos Vicente-Gutierrez ${ }^{1,2,3, *}$ and Juan P. Bolaños ${ }^{1,2,3 \text {, * }}$
}

\author{
${ }^{1}$ Institute of Functional Biology and Genomics (IBFG), University of Salamanca, CSIC, Salamanca, \\ Spain; ${ }^{2}$ Institute of Biomedical Research of Salamanca (IBSAL), Salamanca, Spain; ${ }^{3}$ CIBERFES, \\ Madrid, Spain \\ *For correspondence: barrybiotec@usal.es; ibolanos@usal.es
}

[Abstract] Mitochondrial reactive oxygen species (mROS) are naturally produced signalling molecules extremely relevant for understanding both health- and disease-associated biological processes. The study of mROS in the brain is currently underway to decipher their physiopathological roles and contributions in neurological diseases. Recent advances in this field have highlighted the importance of studying mROS signalling and redox biology at the cellular level. Neurons are especially sensitive to the harmful effects of excess mROS while astrocytic mROS have been shown to play a relevant physiological role in cerebral homeostasis and behaviour. However, given the complexity of the brain, investigating mROS formation in a specific cell-type in adult animals is methodologically challenging. Here we propose an approach to specifically assess mROS abundance in astrocytes that combines i) a targeting strategy based on the use of adeno-associated virus (AAV) vectors expressing the green fluorescent protein (GFP) under an astrocyte (glial fibrillary acidic protein or GFAP) promoter, along with, ii) a robust and widely extended protocol for the measurement of mROS by flow cytometry using commercial probes. The significance of this work is that it allows the selective study of astrocytic mROS abundance by means of easily accessible technology.

Keywords: Reactive Oxygen Species (ROS), Astrocyte, Mitochondria, Adeno-associated virus (AAV) vectors, Oxidative stress, Redox biology, Brain, Neurodegeneration

[Background] Oxidative damage is associated with the aetiology of many diseases, including neurodegenerative disorders given that the brain is an exceptionally vulnerable tissue to oxidative stress as well as to age-related alterations (Cobley et al., 2018; Mattson and Arumugam, 2018). Yet there are still important gaps in the understanding of reactive oxygen species (ROS) pathophysiology in the brain. The deleterious effects associated with ROS in situations of redox stress are in contrast to the increasing evidence suggesting that physiological processes are also fine-tuned by ROS (D'Autréaux and Toledano, 2007; Holmström and Finkel, 2014; Hopkins, 2016a and 2016b). In fact, the clinical application of ROS as signalling molecules is still far away from being an effective solution as a translational therapy (Kamat et al., 2008; Carvalho et al., 2017). Thus, the success of antioxidant therapies depends on considering aspects of redox biology such as its pleiotropism or its cellular and subcellular origin (Juránek et al., 2013; Zhang et al., 2016). In vitro studies using brain cells, principally neurons and astrocytes, have been essential for understanding the highly distinctive biology of these cells. In this sense, it has been 
shown how these brain cells present different molecular specialization, highlighting the importance of crosstalk during neuron-astrocyte coupling which ensures brain bioenergetic and redox homeostasis (Fernandez-Fernandez et al., 2012; Bolaños, 2016). Regarding the study of ROS in brain cells, there is recent evidence indicating that the levels of mitochondrial reactive oxygen species (mROS) are immensely greater-about one order of magnitude-in astrocytes than in neurons (Lopez-Fabuel et al., 2016). This finding reiterates the relevance of considering the cellular origin of neural ROS as an important new factor in the study of redox biology in the brain.

To address this issue, we have reported the use of a new method to quantify the levels of mROS in adult mice astrocytes ex vivo, independently of neurons (Vicente-Gutierrez et al., 2019). This methodology has allowed us to demonstrate an effective and specific down-modulation of astrocytic mROS in a transgenic mouse model expressing a mitochondrial-tagged form of catalase (mitoCAT or mCAT) (Vicente-Gutierrez et al., 2019). In this particular study, we showed that astrocytic mROS has an impact on neuronal function and survival by regulating bioenergetics and redox metabolism. Thus, by decreasing endogenous levels of astrocytic mROS, we found that astrocytic mROS may modulate glucose utilization and neuronal function in behaving mice (Vicente-Gutierrez et al., 2019). Using the methodology that we herein describe, we postulated that endogenous mROS in astrocytes play a physiological role in the maintenance of brain homeostasis. Furthermore, in this same study (VicenteGutierrez et al., 2019), we were able to characterize a novel conditional mCAT mouse that could be useful for testing the implications of mROS in a desired specific tissue type or pathological model.

Since the study of ROS depends not only on concentration but also on its spatiotemporal distribution, real-time imaging of ROS, possibly in vivo, has become necessary in order for scientists to determine which of their biological activities may present a potential for clinical translation. However, this objective is still unachievable owing to current available techniques (Maulucci et al., 2016). Here, we describe in further detail the protocol previously used in Vicente-Gutierrez et al. (2019) to measure mROS abundance, specifically superoxide anion-abundance in astrocytes ex vivo. Although there are different probes commercially available, the most widely used is MitoSOX ${ }^{\mathrm{TM}}$. The MitoSOX Red mitochondrial superoxide indicator enters into mitochondria, where it accumulates in response to the mitochondrial membrane potential $\left(\Delta \Psi_{\mathrm{m}}\right)$ and becomes oxidized. This probe has been widely used in astrocytes in culture (Sheng et al., 2013; Angelova et al., 2015) and in cultured hippocampal sections (Ishii et al., 2017). MitoSOX fluorescence intensity is commonly assessed by microscope imaging or flow cytometry. As mentioned above, this fluorescent dye requires an active mitochondrial membrane potential to enter into mitochondria. This implies that to measure mROS, it is necessary to use live cells and to determine, in parallel, the $\Delta \Psi_{\mathrm{m}}$ in order to confirm that differences in the MitoSOX signal are independent of $\Delta \Psi_{\mathrm{m}}$. However, this fact presents a challenge when working with fixed-cells. For instance, this exclude the use of imaging techniques like immunohistofluorescence in fixed-cells which, in addition, to study individual cellular phenomena required the use of different cell markers. To overcome the cell origin problem, others have used genetically encoded probes to assess redox histology in mouse (Fujikawa et al., 2016). Thus, immunohistochemical strategies still offer a suitable approach for detecting the footprints of redox stress. Nowadays, two-photon laser scanning microscopy has become an interesting 
tool for studying cellular parameters in awake mice, in vivo. However, this technology is not easily accessible in every laboratory and is limited to studying superficial brain areas (Pérez-Alvarez et al., 2013; Wang et al., 2017). Another drawback to this technique is the large amount of time required for sample preparation and for obtaining replicas. In contrast, the protocol that we will describe is relatively easy, accessible, fast and robust.

The ability of certain adeno-associated virus (AAV) vectors to cross the blood-brain barrier after intravenous injection makes it possible to obtain transgene expression in brain cells. The AAV-PHP.eB capsid is one of the serotypes that is able to efficiently transduce the central nervous system (Chan et al., 2017) and is useful for many applications. For instance, we have used it to express the green fluorescent protein (GFP) under the control of the astrocyte-specific glial fibrillary acidic protein (GFAP) short promoter (gfa-ABC ${ }_{1} D$ ) (Lee et al., 2008). Astrocytes were specifically tagged in vivo by infecting mice intravenously through the retro-orbital venous sinus with the AAV-PHP.eB-gfa-ABC ${ }_{1} D-G F P$ construct. Then, we obtained a single brain cell suspension and adapted a well-known protocol to measure MitoSOX by flow cytometry. Therefore, this protocol combines the use of accessible technology like flow cytometry with the use of commercial probes. Accordingly, another advantage to this approach is its versatility, since it is also useful for assessing other cell-specific processes in brain function. By using other brain cell-specific promoters to target neurons, microglia or oligodendrocytes, for instance, the same phenomenon could be measured independently or simultaneously in different cell types. Moreover, this protocol allows the same sample to be analysed using different commercially available probes to measure different biological processes. Finally, this tool is greatly useful to study other cellular and subcellular phenomena and can be used at different time points during model lifespan as well as different tissue types. Overall, we believe that this straight-forward protocol for measuring mROS in adult astrocytes is beneficial for studying redox biology in vivo at the cellular level in the brain.

\section{Materials and Reagents}

\section{A. Anaesthesia}

1. Mice C57BL/6J

2. Sevofluorane (Sevorane ${ }^{\circledR}$ )

3. Oxygen $\left(\mathrm{O}_{2}\right)$ Purity: $99.995 \%$ (Air Liquide, Alphagaz ${ }^{\mathrm{TM}}$ )

4. Nitrous oxide $\left(\mathrm{N}_{2} \mathrm{O}\right)$ (Air Liquide, Alphagaz ${ }^{\mathrm{TM}}$, CAS no.: 010024-97-2)

B. AAVs injection

1. High level surface disinfectant Rely+On ${ }^{\mathrm{TM}}$ Virkon $^{\circledR}\left(\right.$ DuPont $\left.^{\circledR}\right)$

2. Barrier and non-filtered pipette Tips (Art tips, Thermo Scientific)

3. Insulin syringes BD Micro-Fine + Demi $0.3 \mathrm{ml}, 30$ Gauge, $8 \mathrm{~mm}$ (Becton Dickinson, catalog number: 324826 )

4. AAVs. To target astrocytes in this protocol we use AAV-PHP.eB-gfa-ABC ${ }_{1} D-G F P$ construct

5. Pluronic F-127 (Sigma-Aldrich, catalog number: P2443-2506) 
6. Phosphate buffered saline (PBS) (Gibco ${ }^{\mathrm{TM}}$, catalog number: 70011044)

7. Parafilm paper (Bemis, PM-996, USA)

C. Brain single-cell suspension

1. Microsurgical scissor (Fine Science Tools, catalog number: 91501-09)

2. Scalpel blade ( $N^{\circ} 24$, KRAPE S.A., catalog number: BS EN 27740)

3. Fetal bovine serum (FBS) (Gibco ${ }^{\mathrm{TM}}$, catalog number: 10270)

4. Bovine serum albumin (Sigma-Aldrich)

5. DNase I (Roche Diagnostics, catalog number: 10104159001)

6. Trypsin (Sigma-Aldrich, catalog number: T4799)

7. $\mathrm{NaCl}$ (Merck, catalog number: 106404)

8. $\mathrm{KCl}$ (Merck, catalog number: 104936)

9. $\mathrm{MgSO}_{4} \cdot 7 \mathrm{H}_{2} \mathrm{O}$ (Merck, catalog number: 5886 )

10. $\mathrm{NaHCO}_{3}$ (Sigma, catalog number: 6329)

11. $\mathrm{NaH}_{2} \mathrm{PO}_{4} \cdot 2 \mathrm{H}_{2} \mathrm{O}$ (Merck, catalog number: 106345)

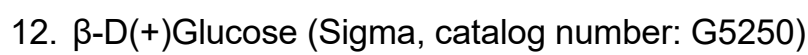

13. Phenol red. (Sigma, catalog number: P5530)

14. $\mathrm{KH}_{2} \mathrm{PO}_{4}$ (Merck, catalog number: 4873 )

15. HEPES (Sigma, catalog number: H3375)

16. $\mathrm{CaCl}_{2} \cdot 2 \mathrm{H}_{2} \mathrm{O}$ (Merck, catalog number: 102381)

17. Earle's Balanced Salt Solution (EBSS) buffer (see Recipes)

18. HBSS buffer (see Recipes)

19. Disaggregation solution (see Recipes)

20. Resuspension solution (see Recipes)

D. Mitochondrial ROS measurement

1. MitoSOX ${ }^{\mathrm{TM}}$ Red Mitochondrial Superoxide Indicator (Molecular Probes, Inc., Invitrogen ${ }^{\mathrm{TM}}$, Thermo Fisher, catalog number: M36008)

\section{Equipment}

A. Anaesthesia procedure and AAVs handling

1. Automatic pipettes (Gilson's PIPETMAN ${ }^{\mathrm{TM}}$ )

2. Microcentrifuge (Eppendorf, model: Centrifuge 5424)

3. Vortex (Scientific Industries, Inc., model: Vortex Genie 2)

4. Anaesthesia system composed by a gas distribution column (Hersill H-3, Spain) and a Vaporizer (InterMed Penlons Sigma Delta) (Figure 1)

5. Class II cabinet equivalent to Telstar Bio II Advance certified according EN-12469-2000 


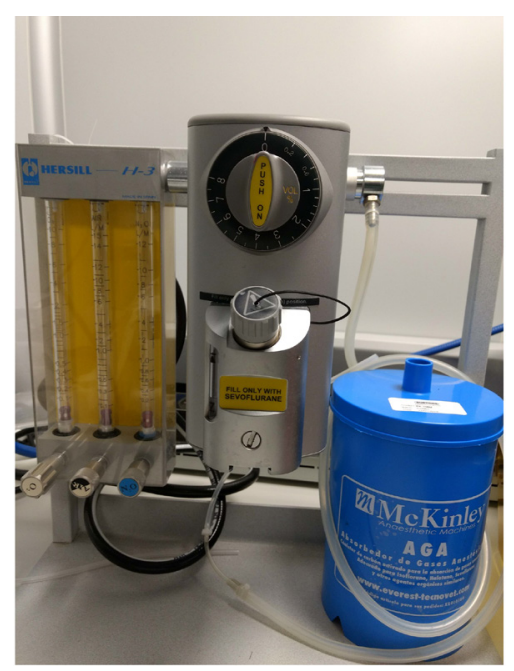

Figure 1. Anaesthesia system

B. Mitochondrial ROS measurement

1. FACSCalibur ${ }^{\mathrm{TM}}$ Flow Cytometer (Becton Dickinson, catalog number: 342975), equipped with a $15 \mathrm{~mW}$ argon laser

2. Centrifuge adapted for Flow cytometry tubes (Eppendorf, model: Centrifuge $5810 \mathrm{R}$ )

\section{Software}

1. BD CellQuest ${ }^{\mathrm{TM}}$ Pro version 5.2 (Becton, Dickinson \& Company, BD Biosciences)

2. FlowJo X 10.0.7r2 (FlowJo, LLC, Becton, Dickinson \& Company)

\section{Procedure}

A. Protocol for the intravenous injection of the adeno-associated virus (AAV) through the retro-orbital venous sinus (Figure 2)

Here, we describe the experimental method designed by Vicente-Gutierrez et al. (2019) using the AAV-PHP.eB-gfa-ABC ${ }_{1} D-G F P$ construct. This protocol is detailed to specifically express a green fluorescent protein (GFP) in the astrocytes of adult mice. However, this approach can be useful to target others cell and tissue types. In that case, using capsids or cell-specific promoters different to AAV-PHP.eB and gfa-ABC $\mathrm{C}_{1} \mathrm{D}$, respectively, it will be necessary to validate considering aspects like the efficient number of $A A V s$ units, route of administration for the injection or mice age. 


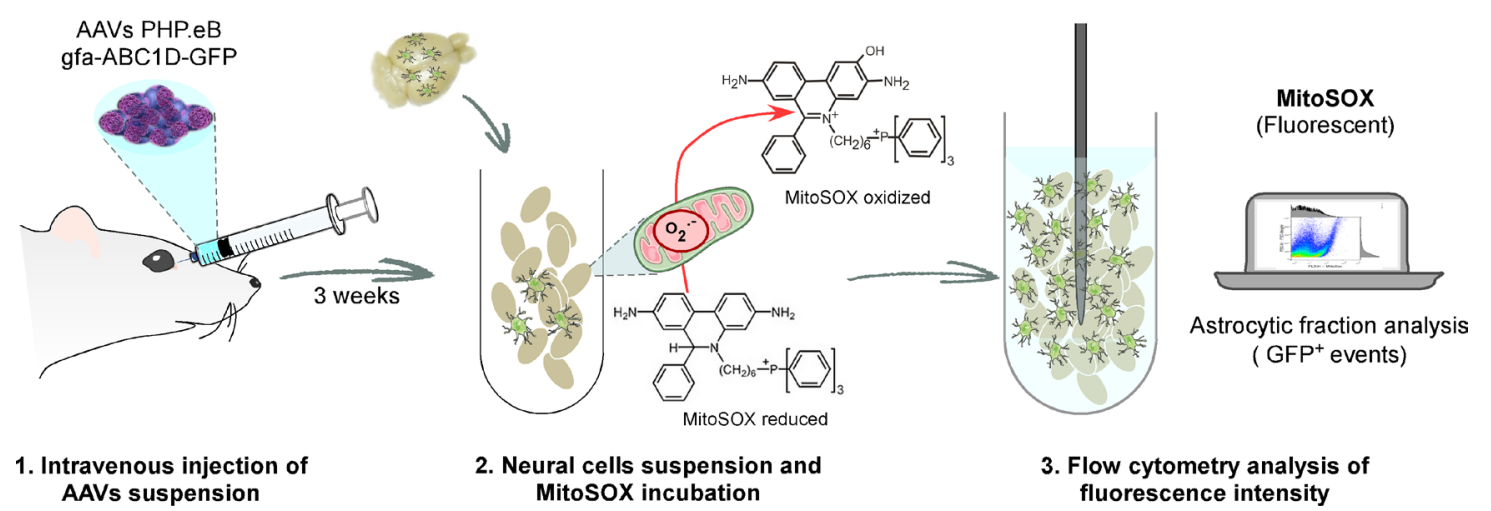

Figure 2. Schematic representation of the protocol to distinguish astrocytes from other neural populations isolated from adult mice by flow cytometry after injection of astroglial AAVs (AAV-PHP.eB-gfa-ABC 1 D-GFP)

1. Prepare a unique AAVs suspension for all mice employed.

Notes:

i. Particle aggregation is a common problem due to a highly concentrated AAV suspension. This phenomenon could be minimized by increasing the ionic strength of the solution where the AAVs are suspended. To do so, add a non-ionic surfactant like pluronic F-127 to PBS solution (Penaud-Budloo et al., 2018). Use PBS solution containing $0.001 \%$ Pluronic F-127 as vehicle solution as well as for the dilutions of AAV suspensions.

ii. Nextstep by step protocol refers to the use of a single AAV construct. However, we recommend using two types of control conditions, namely: 1) mice injected with the vehicle solution (PBS $+0.001 \%$ Pluronic F-127) and 2) mice injected with an empty AAV construct (without GFP) prepared in the same vehicle solution at the desired viral concentration. Both control mice will help to eliminate any possible unwanted responses after infection, as well as to easily distinguish GFP expression in the AAV-GFP-injected mice.

iii. All manipulation should be done within a protective class II laminar flow cabinet. Products in contact with the AAVs must be decontaminated with Rely+On ${ }^{T M}$ Virkon $^{\circledR}$.

a. Calculate the number of mice to be injected on the same day and take the final volume of the AAV suspension needed for all of them. Each adult mouse is injected with $50 \mu \mathrm{l}$ of AAV suspension. Hence, the total volume of AAV suspension is calculated as $V_{T}=50 \mu \mathrm{l}$ the number of injected mice.

b. Prepare $5 \times 10^{10}$ viral genomes of AAV-PHP.eB-gfa-ABC ${ }_{1}$ D-GFP per adult mouse in vehicle solution (PBS $+0.001 \%$ Pluronic F-127). This concentration is based on our previous experience, although it is advisable to check the expression of the protein of interest in the target tissue for each AAV serotype and/or which each promoter. It should be taken into account that the volume of $A A V s$ suspension $\left(V_{A A V}\right)$ may vary according to the dilution of the 
stock of the concentrated AAV suspension.

c. Add the vehicle solution (PBS $+0.001 \%$ Pluronic F-127) to the to the AAVs suspension $\left(V_{A A V}\right)$ in a microcentrifuge tube using a volume equal to $\left(V_{T-} V_{A A V}\right)$ to obtain a volume that will permit $50 \mu \mathrm{l}$ to be injected into each adult mouse. This ensures the desired working dilution of $5 \times 10^{10}$ viral genomes per $50 \mu \mathrm{l}$ of AAVs suspension.

d. Vortex the microcentrifuge tube with the vehicle+AAVs suspension mixture at a working dilution for $10-15 \mathrm{~s}$.

e. Centrifuge the tube for $20 \mathrm{~s}$ at full speed $(\sim 21,000 \times g)$ in a microcentrifuge to recover the total amount of the prepared AAVs suspension.

f. Prepare individual single-use 30 Gauge syringes containing $50 \mu \mathrm{l}$ of the AAVs suspension for each mouse.

i. Use an automatic pipette to ensure accuracy when loading each syringe.

ii. Homogenize by repetitive pipetting ( $\sim 9$ times) before loading each syringe with the $50 \mu \mathrm{l}$ aliquot.

Note: Avoid the formation of bubbles when loading the syringes. An easy way to accurately load the syringes without creating bubbles is to prepare a $50 \mu \mathrm{l}$ drop on a piece of parafilm after homogenization. Then, load each syringe from the parafilm with the bevel down.

2. Mice should be briefly anaesthetized with sevofluorane at $6 \%$ for initiation followed by $\sim 3 \%$ for maintenance in air with supplemented $\mathrm{O}_{2}$ and $\mathrm{NO}_{2}(0.4$ and $0.8 \mathrm{~L} / \mathrm{min}$, respectively) with a gas distribution column and a vaporizer.

Note: Sevoflurane should be administered by a professional trained in the administration of anaesthesia. Respiration should be supervised during sevoflurane administration, and the dose should be reduced upon signs of muscle rigidity.

3. Inject a $50 \mu \mathrm{l}$ aliquot into each anaesthetized animal through the retro-orbital venous sinus and return each one to its home cage (see Yardeni et al., 2011 for specific advice and considerations of this intravenous route of administration).

Note: This route of administration requires specific training. To avoid forcing or making pressure trough eye orbit, the needle is carefully introduced, bevel down, at an angle of approximately $30^{\circ}$, into the medial canthus. Often a small drop of blood is observed at the injection site after administration, which can be cleaned using medical gauze. The total duration of the procedure per mouse is less than 10 min, including injection, anaesthesia and recovery from the effects of the narcotic.

4. Wait three weeks post-injection after the AAV construct has time to be expressed. Then, the astrocyte population of interest can be selected using fluorescent flow cytometry.

Note: Any AAV-containing waste, including bedding and mice faeces, should be collected and placed into biohazard bags during the first week. The waste should then be autoclaved to inactive the AAV. After injection, all mice should be kept in quarantine for at least 3 weeks to avoid possible immune responses, as well as to allow for transgene expression. 
B. Protocol for measuring the mitochondrial reactive oxygen species (mROS) in brain single cell suspensions

This should be performed three weeks after infection with the AAV-PHP.eB-gfa-ABC 1 D-GFP construct expressing GFP in the astrocytes of adult mice. Follow the steps of the protocol described below for extracting the brain and preparing single cell suspension for measuring mROS using the fluorescent commercial probe MitoSOX (Lopez-Fabuel et al., 2016; Vicente-Gutierrez et al., 2019).

\section{Protocol for obtaining single cell suspension of brain tissue:}

1. Sacrifice the mice using cervical dislocation avoiding the use of any asphyxiation method that could interfere with ROS production and bias the subsequent ROS measurement.

2. Extract the brain and, if necessary, isolate the brain regions of interest.

3. At room temperature (RT), rinse the brain tissue in PBS and immerse it in $1 \mathrm{ml}$ of EBSS solution (Recipe 1).

4. Prepare a single-cell suspension from the brain tissue following an enzymatic and mechanical disaggregation procedure (originally described by Almeida and Medina, 1998):

a. Aspirate the total amount of EBSS solution.

b. Cut the brain or brain section using microsurgical scissors or a scalpel blade to obtain $\sim 3 \mathrm{~mm}^{2}$ pieces.

c. Add $500 \mu \mathrm{l}$ of the dissociation solution (Recipe 2) for a whole brain or scale the volume accordingly for smaller pieces.

d. Incubate at $37^{\circ} \mathrm{C}$ for $10 \mathrm{~min}$ with shaking to allow a mild enzymatic dissociation to occur.

e. Neutralize trypsinization with $10 \%$ fetal bovine serum (FBS) to halt the enzymatic dissociation.

f. Centrifuge the tissue ( $5 \mathrm{~min}, 500 \times \mathrm{g}$ ) and discard the supernatant.

g. Add $1 \mathrm{ml}$ of resuspension solution (Recipe 3) and resuspend the pellet using a Pasteur pipette and do a repetitive pipetting (10-15 times) to get a smooth mechanical disaggregation.

h. Leave the suspension to settle for $5 \mathrm{~min}$ and then transfer the supernatant (containing single cells) to a new tube.

i. Add an additional $1 \mathrm{ml}$ of resuspension solution to the remaining pellet and repeat the homogenising process (Steps B4g-B4h) to obtain a final volume of $\sim 2 \mathrm{ml}$ of a single-brain cell suspension (repeat once more if necessary).

\section{Measurement of Mitochondrial ROS abundance:}

5. Split each sample into 4 aliquots: one will be used as a negative control for MitoSOX staining and the other three as test sample technical replicates. The negative control should be resuspended in an appropriate volume ( $1 \mathrm{ml}$ per $10^{6}$ brain cells) of HBSS solution (Recipe 4). The following steps refer to the treatment of the test samples. 
6. Incubate the adult brain cell suspension (use at least 3 technical replicates per sample) together with the fluorescent probe MitoSOX.

a. Prepare MitoSOX working solution; $3 \mu \mathrm{M}$ MitoSOX reagent in HBSS buffer (Recipe 4).

b. Centrifuge the brain cell suspension to obtain a pellet ( $5 \mathrm{~min}, 500 \times \mathrm{g}$ ).

c. Resuspend the pellet by gently and manually shaking the tubes after adding $1 \mathrm{ml}$ of MitoSOX working solution at approximately $10^{6}$ brain cells.

Note: Count cells to adapt the final volume of MitoSOX solution to the total number of cells.

d. Incubate the cells for $30 \mathrm{~min}$ at $37^{\circ} \mathrm{C}$ protected from light, according to the manufacture's protocol.

e. After incubation, wash cells once with $2 \mathrm{ml}$ of warm HBSS buffer.

f. Centrifuge the brain-cell suspension to remove any excess MitoSOX ( 5 min, $500 \times g$ ).

g. Resuspend in HBSS buffer using an appropriate volume to obtain a single-cell suspension to be analysed in the flow cytometer.

Note: An appropriate volume per replicate derived from a whole brain is $500 \mu l$, scale the volume accordingly for smaller areas. This volume allows a final dilution to asses less than 300 events/second tolerable to FACScalibur, considering an 'event' a singular cell.

h. For flow cytometry, use approximately $510 \mathrm{~nm}$ excitation and a fluorescence channel able to detect the $580 \mathrm{~nm}$ emission of oxidized MitoSOX reagent (FL-3 in BD FACSCalibur ${ }^{\mathrm{TM}}$ flow cytometer).

7. Flow cytometry:

Note: Flow cytometry requires previous training to set compensation of signal fluorescence and to adapt the parameters of the flow cytometer to brain cells size and complexity.

a. Use tubes containing the control samples to calibrate the desired voltage adjustments for each channel used to detect single or combined stains. The compensation of each channel avoids the interference of fluorescence signals in different channels. The control samples used for the purpose of calibration in this protocol are:

Non-stained cells. Cells without any stain (no MitoSOX) derived from vehicle-injected mice (no GFP).

Cells exclusively positive for MitoSOX channel (FL-3 in BD FACSCalibur ${ }^{T \mathrm{M}}$ flow cytometer). Cells derived from vehicle-injected mice incubated with MitoSOX.

Cells exclusively positive for GFP channel (FL-1 in BD FACSCalibur ${ }^{\mathrm{TM}}$ flow cytometer).

Cells derived from any mice injected with AAVs-GFP (AAV-PHP.eB-gfa-ABC ${ }_{1} D-G F P$ ) without incubation with MitoSOX.

Note: As mentioned in Steps B5, it is advisable to split one replicate per sample (mouse/brain area) to obtain a non-stained negative control sample to eliminate unwanted measurements in the MitoSOX channel.

b. Finally, test samples should be assessed for both previously calibrated channels:

i. First, set the voltage in forward (FSC: cell size) and side scatter (SSC: cell complexity) to centre the population and properly visualize the sample in the dot plot $X Y$ graph. 
ii. Calibrate and compensate channels using the control samples (Review StepB7a above).

iii. Once calibrated, pass each tube using the established parameters and record at least $\sim 100,000-200,000$ events (this number may vary depending on the expected percentage of GFP positive population).

\section{Data analysis}

Use FlowJo or similar software to quantify the intensity of MitoSOX signal in the brain samples. First, gate on the cells excluding debris or doublets events looking at forward (size) and side (complexity) scatter scales. Then, measure the thresholds for GFP and MitoSOX signals with negative control samples used to perform standard compensation (Figure 3). Once these limits are established, determine the mean of the fluorescent intensity values for the events included in the gate of interest.

It is advisable to use at least three technical replicates for each sample to obtain a robust mean for each biological replicate. Results are expressed as arbitrary units of signal intensities.

Figure 3 represents the workflow used in the flow cytometry analysis, which served to show that mice expressing a mitochondrial-tagged isoform of catalase (mCAT) in astrocytes present lower levels of astrocytic mROS (see Vicente-Gutierrez et al., 2019). 

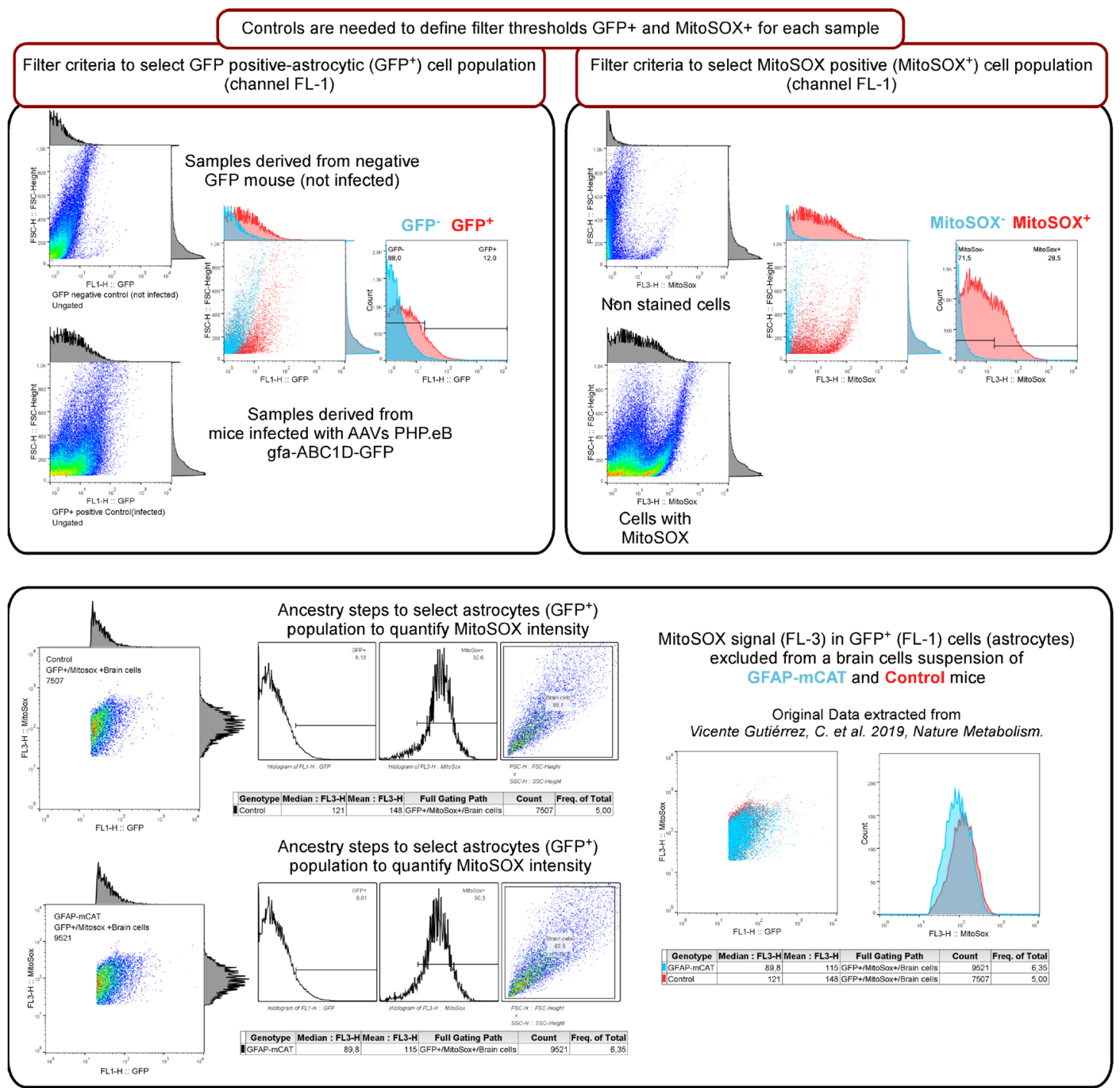

Figure 3. Flow cytometry workflow for brain cells experiments to measure mitochondrial ROS (mROS) (using MitoSOX) exclusively in astrocytes expressing GFP after the infection of mice with AAV-PHP.eB-gfa-ABC ${ }_{1}$ D-GFP (original data is contained in Vicente-Gutierrez et al., 2019).

\section{$\underline{\text { Recipes }}$}

Take into account that some of these solutions are commercially available.

1. Earle's Balanced Salt Solution (EBSS) buffer

$116 \mathrm{mM} \mathrm{NaCl}$

\section{$5.4 \mathrm{mM} \mathrm{KCl}$}

$1.5 \mathrm{mM} \mathrm{MgSO}_{4}$

$26 \mathrm{mM} \mathrm{NaHCO}_{3}$

$1.01 \mathrm{mM} \mathrm{NaH}_{2} \mathrm{PO}_{4} \cdot 2 \mathrm{H}_{2} \mathrm{O}$

$4 \mathrm{mM}$ glucose

Phenol red $10 \mathrm{mg} / \mathrm{L} \mathrm{pH} 7.2$ 
2. Dissociation/Disaggregation solution

EBSS solution supplemented with bovine serum albumin $0.3 \%(\mathrm{p} / \mathrm{v})$ (Sigma-Aldrich)

DNase I $20 \mu \mathrm{g} / \mathrm{ml}$ (Roche)

Trypsin 0.025\% p/v (Sigma-Aldrich) (Almeida and Medina, 1998)

3. Resuspension solution

EBSS solution supplemented with supplemented with bovine serum albumin $0.3 \%(\mathrm{p} / \mathrm{v})$ (SigmaAldrich)

DNase I $20 \mu \mathrm{g} / \mathrm{ml}$ (Roche) (Almeida and Medina, 1998)

4. HBSS buffer

$134.2 \mathrm{mM} \mathrm{NaCl}$

$5.26 \mathrm{mM} \mathrm{KCl}$

$0.43 \mathrm{mM} \mathrm{KH}_{2} \mathrm{PO}_{4}$

$4.09 \mathrm{mM} \mathrm{NaHCO}_{3}$

$0.33 \mathrm{mM} \mathrm{Na}_{2} \mathrm{HPO}_{4} \cdot 2 \mathrm{H}_{2} \mathrm{O}$

$5.44 \mathrm{mM}$ glucose

$20 \mathrm{mM}$ HEPES

$20 \mathrm{mM} \mathrm{CaCl} 2 \cdot 2 \mathrm{H}_{2} \mathrm{O}$

$\mathrm{pH} 7.4$

\section{Acknowledgments}

We acknowledge the technical assistance and mice care carried out by M. Carabias-Carrasco, L. Martin, E. Prieto-Garcia and M. Resch from the University of Salamanca as well as the laboratory of G. Bonvento at the Molecular Imaging Center (MIRCen), CNRS UMR 9199, Université Paris, France for providing the AAVs and helpful advice. We would also like to thank Nicoló Bonora and Daniel Jimenez-Blasco who participated in the experimental work involving this procedure.

This work was funded by MINECO (SAF2016-78114-R), Instituto de Salud Carlos III (CB16/10/00282), NIH/NIDA (1R21DA037678-01), Ayudas Equipos Investigación Biomedicina 2017 Fundación BBVA, Fundación Ramón Areces and Junta de Castilla y León (Escalera de Excelencia CLU-2017-03). This protocol was originally described by Vicente-Gutierrez et al. (2019).

\section{Competing interests}

The authors declare no competing interests.

\section{Ethics}

All animal procedures we performed according to the European Union Directive 86/609/EEC and Recommendation 2007/526/EC, regarding the protection of animals used for experimental and other 
Please cite this article as: Vicente-Gutierrez and Bolaños, (2020). An ex vivo Approach to Assess Mitochondrial ROS by Flow Cytometry in AAV-tagged

scientific purposes, enforced in Spanish legislation under the directive RD1201/2005. All protocols were approved by the Bioethics Committee of the University of Salamanca.

\section{References}

1. Almeida, A. and Medina, J. M. (1998). A rapid method for the isolation of metabolically active mitochondria from rat neurons and astrocytes in primary culture. Brain Res Brain Res Protoc 2(3): 209-214.

2. Angelova, P. R., Kasymov, V., Christie, I., Sheikhbahaei, S., Turovsky, E., Marina, N., Korsak, A., Zwicker, J., Teschemacher, A. G., Ackland, G. L., Funk, G. D., Kasparov, S., Abramov, A. Y. and Gourine, A. V. (2015). Functional oxygen sensitivity of astrocytes. J Neurosci 35(29): 10460-10473.

3. Bolaños, J. P. (2016). Bioenergetics and redox adaptations of astrocytes to neuronal activity. $J$ Neurochem 139 Suppl 2: 115-125.

4. Carvalho, A. N., Firuzi, O., Gama, M. J., Horssen, J. V. and Saso, L. (2017). Oxidative stress and antioxidants in neurological diseases: is there still hope? Curr Drug Targets 18(6): 705-718.

5. Chan, K. Y., Jang, M. J., Yoo, B. B., Greenbaum, A., Ravi, N., Wu, W. L., Sanchez-Guardado, L., Lois, C., Mazmanian, S. K., Deverman, B. E. and Gradinaru, V. (2017). Engineered AAVs for efficient noninvasive gene delivery to the central and peripheral nervous systems. Nat Neurosci 20(8): 1172-1179.

6. Cobley, J. N., Fiorello, M. L. and Bailey, D. M. (2018). 13 reasons why the brain is susceptible to oxidative stress. Redox Biol 15: 490-503.

7. D'Autréaux, B. and Toledano, M. B. (2007). ROS as signaling molecules: mechanisms that generate specificity in ROS homeostasis. Nat Rev Mol Cell Biol 8(10): 813-824.

8. Fernandez-Fernandez, S., Almeida, A. and Bolanos, J. P. (2012). Antioxidant and bioenergetic coupling between neurons and astrocytes. Biochem J 443(1): 3-11.

9. Fujikawa, Y., Roma, L. P., Sobotta, M. C., Rose, A. J., Diaz, M. B., Locatelli, G., Breckwoldt, M. O., Misgeld, T., Kerschensteiner, M., Herzig, S., Muller-Decker, K. and Dick, T. P. (2016). Mouse redox histology using genetically encoded probes. Sci Signal 9(419): rs1.

10. Holmström, K. M. and Finkel, T. (2014). Cellular mechanisms and physiological consequences of redox-dependent signaling. Nat Rev Mol Cell Biol 15(6): 411-421.

11. Hopkins, R. (2016a). Hydrogen peroxide in biology and medicine: An overview. Reactive Oxygen Species 3(7): pp. 26-37.

12. Hopkins, R. (2016b). Superoxide in biology and medicine: An overview'. Reactive Oxygen Species 1(2): pp. 99-109.

13. Ishii, T., Takanashi, Y., Sugita, K., Miyazawa, M., Yanagihara, R., Yasuda, K., Onouchi, H., Kawabe, N., Nakata, M., Yamamoto, Y., Hartman, P. S. and Ishii, N. (2017). Endogenous reactive oxygen species cause astrocyte defects and neuronal dysfunctions in the hippocampus: a new model for aging brain. Aging Cell 16(1): 39-51. 
Please cite this article as: Vicente-Gutierrez and Bolaños, (2020). An ex vivo Approach to Assess Mitochondrial ROS by Flow Cytometry in AAV-tagged

14. Juránek, I., Nikitovic, D., Kouretas, D., Hayes, A. W. and Tsatsakis, A. M. (2013). Biological importance of reactive oxygen species in relation to difficulties of treating pathologies involving oxidative stress by exogenous antioxidants. Food Chem Toxicol 61: 240-247.

15. Kamat, C. D., Gadal, S., Mhatre, M., Williamson, K. S., Pye, Q. N. and Hensley, K. (2008). Antioxidants in central nervous system diseases: preclinical promise and translational challenges. J Alzheimers Dis 15(3): 473-493.

16. Lee, Y., Messing, A., Su, M. and Brenner, M. (2008). GFAP promoter elements required for region-specific and astrocyte-specific expression. Glia 56(5): 481-493.

17. Lopez-Fabuel, I., Le Douce, J., Logan, A., James, A. M., Bonvento, G., Murphy, M. P., Almeida, A. and Bolanos, J. P. (2016). Complex I assembly into supercomplexes determines differential mitochondrial ROS production in neurons and astrocytes. Proc Natl Acad Sci U S A 113(46): 13063-13068.

18. Mattson, M. P. and Arumugam, T. V. (2018). Hallmarks of brain aging: adaptive and pathological modification by metabolic states. Cell Metab 27(6): 1176-1199.

19. Maulucci, G., Bacic, G., Bridal, L., Schmidt, H. H., Tavitian, B., Viel, T., Utsumi, H., Yalcin, A. S. and De Spirito, M. (2016). Imaging Reactive Oxygen Species-Induced Modifications in Living Systems. Antioxid Redox Signal 24(16): 939-958.

20. Penaud-Budloo, M., Francois, A., Clement, N. and Ayuso, E. (2018). Pharmacology of Recombinant Adeno-associated Virus Production. Mol Ther Methods Clin Dev 8: 166-180.

21. Pérez-Alvarez, A., Araque, A. and Martin, E. D. (2013). Confocal microscopy for astrocyte in vivo imaging: Recycle and reuse in microscopy. Front Cell Neurosci 7: 51.

22. Sheng, W. S., Hu, S., Feng, A. and Rock, R. B. (2013). Reactive oxygen species from human astrocytes induced functional impairment and oxidative damage. Neurochem Res 38(10): 21482159 .

23. Vicente-Gutierrez, C. Nicoló, B., Bobo-Jimenez, V., Jimenez-Blasco, D., Lopez-Fabuel, I., Fernandez, E., Josephine, C., Bonvento, G., Enriquez, Jose A., Almeida, A. and Bolaños, J. P. (2019). Astrocytic mitochondrial ROS modulate brain metabolism and mouse behaviour. Nature Metabolism 1: 201-211.

24. Wang, H., Zhang, R., Bridle, K. R., Jayachandran, A., Thomas, J. A., Zhang, W., Yuan, J., Xu, Z. P., Crawford, D. H., Liang, X., Liu, X. and Roberts, M. S. (2017). Two-photon dual imaging platform for in vivo monitoring cellular oxidative stress in liver injury. Sci Rep 7: 45374.

25. Yardeni, T., Eckhaus, M., Morris, H. D., Huizing, M. and Hoogstraten-Miller, S. (2011). Retroorbital injections in mice. Lab Anim (NY) 40(5): 155-160.

26. Zhang, J., Wang, X., Vikash, V., Ye, Q., Wu, D., Liu, Y. and Dong, W. (2016). ROS and ROSMediated Cellular Signaling. Oxid Med Cell Longev 2016: 4350965. 